\title{
Perspectives on Bayesian Methods and Big Data
}

\author{
Greg M. Allenby • Eric T. Bradlow • Edward I. George • \\ John Liechty $•$ Robert E. McCulloch
}

Published online: 5 June 2014

(C) Springer Science+Business Media New York 2014

\begin{abstract}
Researchers and practitioners are facing a world with ever-increasing amounts of data and analytic tools, such as Bayesian inference algorithms, must be improved to keep pace with technology. Bayesian methods have brought substantial benefits to the discipline of Marketing Analytics, but there are inherent computational challenges with scaling them to Big Data. Several strategies with specific examples using additive regression trees and variable selection are discussed. In addition, the important observation is made that there are limits to the type of questions that can be answered using most of the Big Data available today.
\end{abstract}

Keywords Bayesian statistics $\cdot$ Big Data $\cdot$ Scalable computation

\section{Introduction}

Advances in technology, with regards to computing and communication, have led to an ever increasing amount of data that can provide summaries of our modern life. These summaries can give insights into both overall systems as well as details about individual actors. Almost in parallel, advances in Bayesian inference methods (e.g., the Monte Carlo Markov

\section{G. M. Allenby}

Fisher College of Business, The Ohio State University,

Columbus, OH, USA

\section{E. T. Bradlow $\cdot$ E. I. George}

The Wharton School, University of Pennsylvania, Philadelphia, PA, USA

J. Liechty $(\bowtie)$

Smeal College of Business, The Pennsylvania State University, State

College, PA, USA

e-mail: jc112@psu.edu

R. E. McCulloch

Booth School of Business, University of Chicago, Chicago, IL, USA chain algorithm) have allowed researchers to gain insights about consumers, using more relevant and complex models. One of the current challenges facing the discipline of Marketing Analytics (and many related empirically driven disciplines) is the challenge of scaling (or modifying) existing inference methods to take advantage of this growing source of data and the related increasing computational resources.

The following are perspectives regarding how Bayesian statistical methods can be used effectively in a future that has more and more data and computing resources. In the second section, Bradlow discusses some of the strengths of a Bayesian modeling framework that make it so well suited for Marketing Analytics, namely, its ability to deal with heterogeneity. He then talks in general terms about various strategies that can be used to make Bayesian methods computationally effective with Big Data. In the third section, George and McCulloch build on the theme of providing strategies which make Bayesian methods computationally efficient. They provide two examples, one using a Bayesian Additive Regression Tree (BART) model and the other using an alternative for variable selection on Big Data. In the BART example, they highlight two different strategies for using the Markov Chain Monte Carlo (MCMC) algorithm in a parallel computing environment. In the fourth section, Allenby gives a timely reminder that there are limits to the types of questions that can be answered with this ever increasing Big Data. He points out that most of Big Data simply captures what is happening in the "field" and as a result has limited value for making strategic decisions. He aptly points out that Marketing Analytics ultimately exists to help firms create products "that will be highly valued by consumers, at a price and place, and with a message, that is relevant to their needs," and in order to do this, firms typically need to conduct experiments as opposed to collect market transaction data with its inherent endogeneities and lack of competing "potential solutions." Analysis of Big Data can help with tactical marketing decisions (e.g., helping guide and improve individual consumer decisions), but it will require thoughtful efforts with additional data collection (which 
can be made easier by the same technology that gives us our Big Data) to create the products that will improve the world.

\section{Bayesian Analysis and Big Data, not Bayesian Analysis or Big Data}

Bayesian analyses applied to marketing science problems, since their inception [1], have become increasingly popular due to their ability to capture individual-level customer heterogeneity that "with certainty" exists. Whether it is heterogeneity in responsiveness to undiscounted price, coupons, advertising, distribution channel effectiveness, etc., ignoring these effects, rather than "celebrating heterogeneity," when using these models as decision support tools can lead to a significant misallocation of resources.

Yet, despite their appropriateness for handling the heterogeneity and sparseness (at the customer level) that exists in many marketing data sets, Bayesian methods as commonly applied come with the unfortunate "burden" of significant computational cost. This arises mainly, but not entirely, due to the non-conjugacy of most marketing models of consumer behavior (e.g., the conditional logit model, [14]) with the assumed heterogeneity distribution (e.g., a normal or mixture of normals; [8]) leading to intractable integrals. While MCMC [7] and associated methods "solve" this problem in concept, it does not yield real-time posterior distributions even for small to medium size data sets (e.g., which can be handled in standard software) and whether in real time or not their application to "big data" is questionable. So, what to do?

A number of possible approaches exist which I briefly discuss, recognizing that not applying Bayesian methods to large-scale problems given the recent ability for firms to track individuals purchases, marketing activity, user-generated content [13], survey responses, etc. in one integrated database (cf. Wharton Customer Analytics Initiative, http://www.wharton. upenn.edu/wcai/) would be nonsensical.

First and foremost, most marketing problems are very amenable to sampling where that sampling could be of individual units (customers), time periods, and/or stores/ geographic regions. Certainly, papers that are methodological in nature would not suffer from turning big data into more manageable computational data, and many substantive problems can easily be addressed through short time-series (albeit, not within-customer heterogeneity models like HMMs, [17]), or by running multiple shorter analyses and using metaanalytic methods [23] to combine the results. With increased parallel computing, e.g., Amazon Cloud Services, this has the potential for massively distributed computing followed by very fast real-time computation.

A second stream of research that could allow for Bayesian methods to be applied to big data problems is the polynomial methods that allow the researcher to approximate the likelihood function which is then amenable to closed-form computation. In Bradlow et al. [2], these approximation methods are applied to count data in the form of a negative-binomial distribution. This was subsequently extended by Everson and Bradlow [5] to the Beta-Binomial model and then more generally to choice models [15]. This research is all related to recent research on Variational Bayesian Methods (http://www.variational-bayes.org/; [10]) that provide more general approximation methods.

Finally, while the lore of big data and predictive analytics has led many companies to build expansive CRM systems, much of that data does not provide direct economic value to the firm. This suggests that an increase focusing on statistical sufficiency, very related to the computer science literature on data compression, will become increasingly important; that is, as it becomes easier and easier for firms to collect more data to create big data, firms should spend more and more time thinking about what data to keep.

Thus, while it may seem to many that the choice is Bayesian methods or big data, which ignores many sampling, compression, approximation, and meta-analytic methods that can break the computational problem. Furthermore, as realtime solutions are becoming increasingly important, we should expect further advances to come quickly.

\section{Bayes and Big Data: Innovating BART, Spike-and-Slab Variable Selection and Beyond}

\subsection{The Big Data Challenge to Bayesian Statistics}

The advent of MCMC methods in the early 1990s served to emancipate the implementation of Bayesian analysis. Methods which had been stymied by computation constraints became practical because of advances in computing and storage technology and methods which could exploit them. Today, as the relentless advances of such technology have now led to vast increases in the size and complexity of data, innovations are continually needed to free Bayesian analysis to handle this new big data environment. In this section, we describe recent innovations to two of our earlier MCMC driven approaches, BART and spike-and-slab variable selection. These innovations serve to vastly increase the potential of the underlying Bayesian methods to cope with big data.

\subsection{BART}

BART (Chipman et al. [4], Chipman, George, and McCulloch, henceforth CGM) provides a Bayesian inference for the fundamental model

$$
Y=f(x)+\epsilon, \quad \in \sim N\left(0, \sigma^{2}\right),
$$


where $f$ is unknown. BART is a Bayesian tree-based approach which CGM show to be competitive with modern alternatives in terms of out-of-sample predictive performance.

Some key advantages of the BART approach are the following:

1. Reasonable results are often obtained using the default prior (as opposed to using cross-validation to tune the prior choice).

2. The depth of the trees used to construct the function $f$ is inferred relatively automatically so that the level of interaction between variables can be naturally uncovered.

3. As with other tree-based model, there is no need to specify a set of transformation in the $x$ space.

4. The level of uncertainty is obtained through the usual Bayes posterior.

CGM illustrate a variety of BART applications (including high-dimensional $x$ ) using the implementation of BART in the $\mathrm{R}$ package BayesTree. However, the BayesTree implementation is painfully slow even for "small" data sets with 10,000 observations and unusable for larger data sets. Pratola et al. [19] (henceforth PBART) and Scott et al. [22] (henceforth SBART) consider two simple approaches to BART algorithms which are effective for data sets with a large number of observations using parallel computation. Scott et al. [22] present a general approach to parallel computations for Bayesian models and SBART is just an example.

The basic BART algorithm of CGM is a MCMC approach. The PBART approach is to fully implement a simplified version of the BART MCMC using parallel computation on subsets of the data every time a (conditional) sufficient statistic is needed. The PBART approach requires frequent message passing between machines and is implemented using the Message Passing Interface. PBART argues that BART is ideal for this basic approach to parallel computation as the size of the passed messages can be kept small relative to the overall computational burden. SBART also splits the data across machines but runs a separate MCMC using each data subset and then combines the results using a weighted average. Thus, PBART runs one MCMC while SBART runs $n$ core MCMCs, where $n$ core is the number of cores. The advantage of SBART is that frequent message passing is not needed. SBART is easily implemented (for example, using the parallel package in R).

Figure 1 shows the efficiency of the PBART algorithm using a cluster of machines. On the horizontal axis is the number of observations allocated to each core. On the vertical axis is the efficiency. An efficiency of 1 would mean that the computations are 50 times faster with 50 cores. If you put enough observations (more than 20,000) on a core, the efficiency is up around $90 \%$ which represents a dramatic speedup.
Figure 2 illustrates the SBART approach. Using simulated data, we compare the true $f(x)$ with the estimates. The true $f(x)$ are on the horizontal axis and the estimates are on the vertical axis. A random subset of points is plotted. The estimates from a full BART run are plotted with $\mathrm{a}+$, the SBART estimates are plotted with a solid symbol, and estimates from the separate MCMC runs on the data subsets (or shards) are plotted with a smaller dot.

The estimates from the individual BART MCMC's run on data subsets vary, but the average of these estimates looks just as good as that obtained from the full BART run.

\subsection{Spike-and-Slab Variable Selection}

Spike-and-slab variable selection is an approach to Bayesian variable selection for the normal linear model

$Y=x^{\prime} \beta+\epsilon, \quad \in \sim N\left(0, \sigma^{2}\right)$,

where $\beta$ is an unknown $p$-dimensional vector of regression coefficients. The basic idea is to use a spike-and-slab mixture prior to induce a posterior distribution over the set of $2^{p}$ possible subset models. For this setup, George and McCulloch [9] proposed an approach called stochastic search variable selection (SSVS) which used an MCMC posterior simulation to search for and identify high posterior models. Unfortunately, SSVS quickly becomes impractical with big data as $p$ gets very large because the space of $2^{p}$ possible models becomes too vast.

To increase the effectiveness of spike-and-slab variable selection for big data, a new procedure expectationmaximization variable selection (EMVS) [20] uses an expectation-maximization (EM) algorithm to deterministically search for high posterior modes. EMVS effectively substitutes MCMC simulation from a full posterior with the EM-directed constrained optimization problem of finding a single mode. For large $p$, EMVS can more quickly gravitate towards high posterior modes. In fact, the speed of EMVS allows for enhanced dynamic posterior exploration as hyperparameters are varied. For large $n$, feasible implementation of the EM algorithm is achieved by sampling the data to obtain approximate, but manageable, calculations, and EMVS is emancipating a fundamental Bayesian approach for the big data problems ahead.

\subsection{General Comments}

The development of effective big-data computational approaches for Bayesian inference presents challenges, but fortunately, new approaches are quickly extending Bayes procedures to make them a big-data tool.

Perhaps the most common approaches to big-data Bayes are (i) simply computing a mode, which reduces the problem to an optimization and (ii) exploiting conditional 
Fig. 1 Efficiency of PBART algorithm

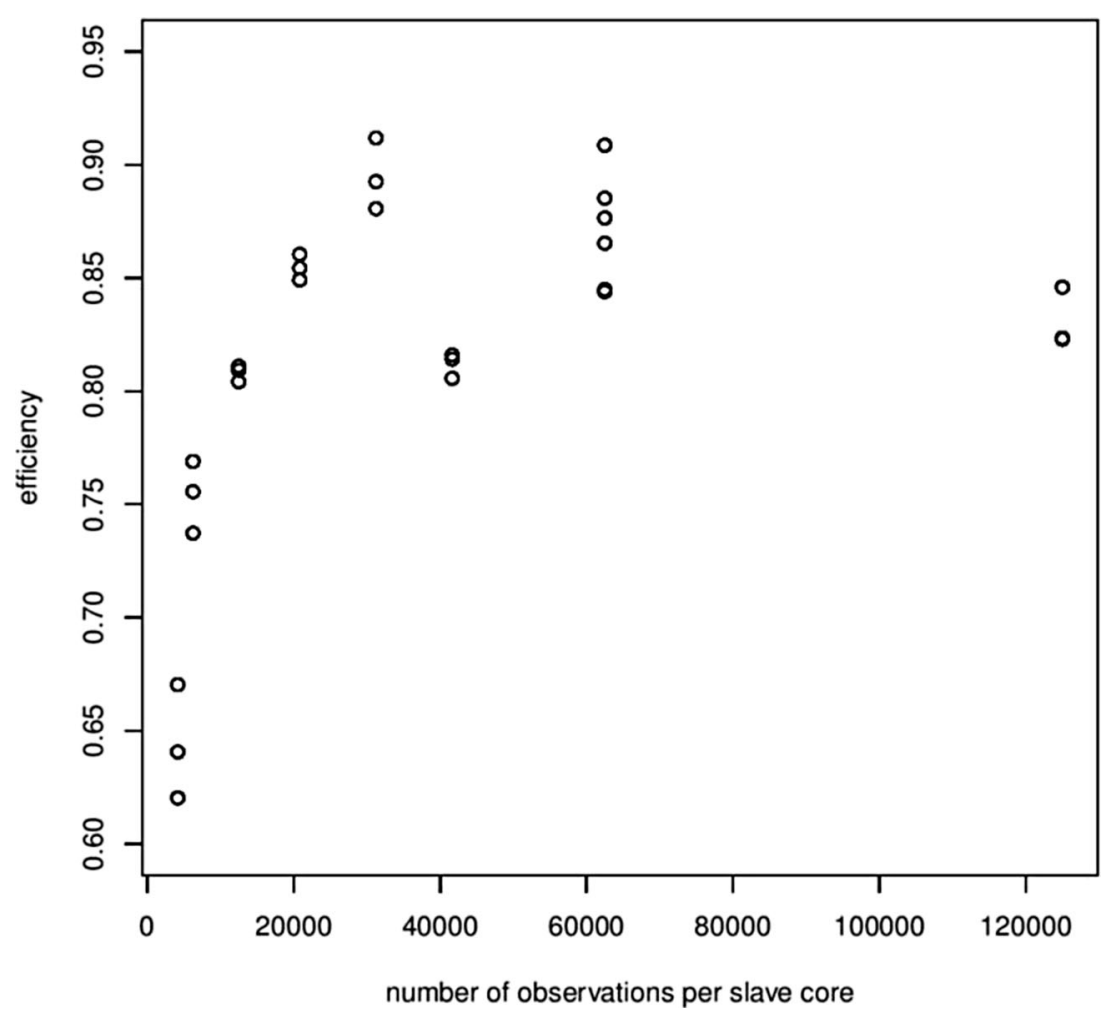

independence in an MCMC to parallelize an MCMC step. However, a variety of methods are being developed. PBART is a parallel computation approach which is very specific to
BART. Indeed, the special form of BART enables a simple distributed data approach not possible for many other Bayesian non-parametric models. More generally, one can
Fig. 2 SBART: sharded fit and fit of shards

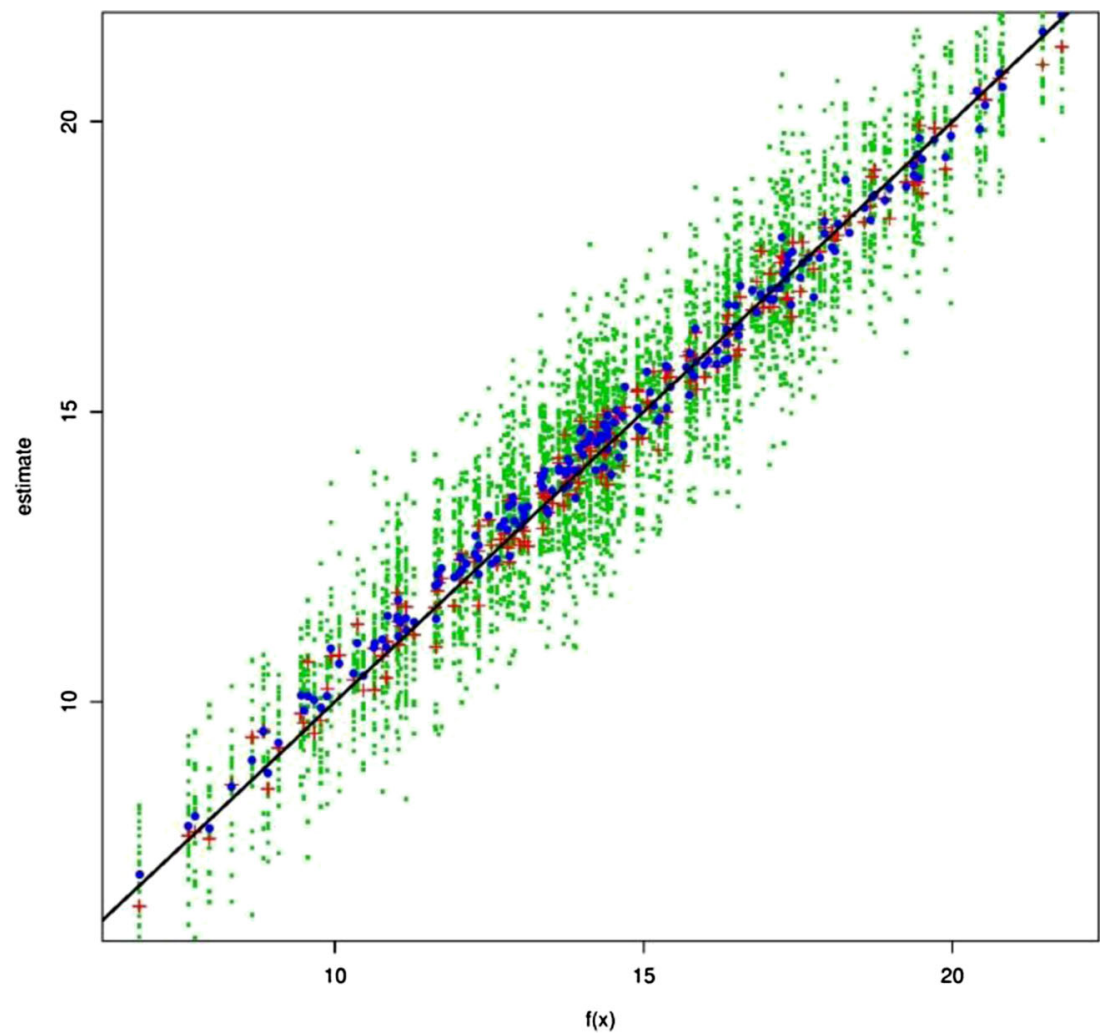


parallelize individual steps within an MCMC algorithm, see for example Tibbits et al. [24]).

In contrast, SBART is an implementation of a very general approach (consensus Monte Carlo) which may be applied to any Bayesian model for exchangeable data. However, the Pratola et al. approach exactly replicates the basic BART algorithm while the Scott et al. approach assumes that the sharded runs explore the same breadth of model space as a full BART run.

In another direction, EMVS uses deterministic search to find posterior modes under spike-and-slab variable selection, bypassing the need for stochastic search. Such speedups will become essential as the number of potential predictors in data continues to explode.

\section{Bayes and Big Data's Impact on Marketing Research}

The Big Data revolution is prompting the collection and archiving of an unprecedented amount of data by firms. The marketing industry is convinced there is gold in Big Data, if only it can be harvested into meaningful insights. Big Data arises from the recording of marketplace transactions of individuals, offering the hope of a more complete understanding of consumption patterns and therefore a better understanding of what makes people tick. Knowing what people want in one category (e.g., baby food) is useful information for crossselling and identifying potential customers in complementary product categories (e.g., diapers, strollers, bedding). Big Data tells us about the responses of individual customers to marketplace offerings across a multitude of product categories, which is useful in many endeavors such as establishing baselines for measuring effect sizes in online pricing and advertising experiments.

However, despite all of the excitement about it, Big Data is deficient on many dimensions. First, it reveals what people want but not why they want it. Need states and associated consumption contexts are not revealed [6], making this type of marketplace data uninformative about how products are effective in providing solutions to consumer problems. In reality, Big Data is huge because of its breath, not because of its depth. It provides large amounts of shallow data that does not reveal the following:

(i) The state of the respondent-were they informed or uninformed about the category? Were they in a rush to purchase? Who was with them? Was the purchase for themselves or someone else?

(ii) The state of the market - we only observe what was purchased, not what else was compared. Big data often lacks information about the prices and availability of competitive products so that constructs like a customer's utility function can be calibrated.
Big Data is also discrete. Choices are recorded as binary events, and when purchase quantities are recorded the amounts lie on a grid defined by the available package sizes [12]. This "indivisibility" creates problems when trying to understand whether people do not make purchases because they do not like the product or because the smallest package size has more of the product than they want to handle (e.g., pastries at Panera). Probably the most striking aspect of Big Data is the prevalence of the number " 0 " in the data. The high proportion of zeros indicates that consumers are goal-directed and resource-conserving in their marketplace behavior. Consumers do not try most of the brands in a product category, do not visit most of the associated websites, do not learn about most of the competitor brands, and do not understand the depth of options because they have limited resources in form of time, money, and attention. At a fundamental level, these zeros are qualitatively different from positive quantities, providing limited information about what is going on in the life of respondents [11]. There are more reasons for not purchasing something than purchasing it, and it is not clear that that the zeros and positive quantities of observed marketplace demand should be treated as alternative outcomes from a multinomial variable.

Additional problems with marketplace data include the presence of self-selection, where there is a complicated story for why respondents are included in an analysis in the first place. Self-selection is not present in a controlled experiment with random assignment to treatment conditions. Similarly, marketplace prices, distribution decisions, advertisement, and product variables are set in anticipation of marketplace responses, making them endogenously determined. This endogeneity is not present in a controlled experiment where the levels of these variables are independently set by the researcher. Little is known about how prices and other marketing variables (i.e., the $4 \mathrm{Ps}$ ) are set in practice, making controls for endogeneity difficult to achieve [18].

The upshot of the deficiencies of Big Data is that they are not very useful for making strategic marketing decisions but can be useful for tactical decisions. Marketers have come to realize this in the use of Big Data for fine-tuning prices and searching for cross-selling opportunities, conditional on most of the 4Ps staying the same, that is, Big Data is not being used to guide major brand reformulation and positioning decisions because these efforts require more in-depth analysis. However, if the distribution channels, advertising and promotional appeals, and the product is relatively fixed, then Big Data can be informative about issues related to fine-tuning the price of a product. Big Data, for example, cannot be used to identify or measure unmet demand because its nature is from demand currently met in the marketplace [3].

A final complexity of Big Data is that it is big - really big. Achieving high parsimony is therefore needed in models employing these data. One way of achieving parsimony is with the use of theory. Economic theory, for example, 
postulates the existence of utility underlying consumer preferences and choices, and the calibration of parsimonious utility functions (e.g., as in discrete choice models) is a fruitful avenue of research in Big Data models. Another approach is the use of random effects model to shore-up information deficiencies due to the shallowness of Big Data [1]. Both approaches help in avoiding the influence of outliers in the data and other contaminants that lead to spurious results in high-dimensional analysis. It has long been recognized in marketing that simpler models lead to more robust predictions, and the use of theory to explain variation in Big Data is valued because of its size.

Since marketing's role is to guide firms on what to produce that will be highly valued by consumers, at a price and place, and with a message that is relevant to their needs, the use of theory and statistical inference tools will remain a cornerstone of marketing analysis. Bayesian methods are particularly useful in this pursuit because of their ability to deal with large, shallow datasets and their ability to produce exact, finite sample inference.

Looking to the future, the Internet has made it possible to engage in research on selective issues and topics at a very low cost. Google consumer surveys, for example, allow researchers to pose a small number of questions to people on the web for as little as US $\$ 0.10$ per respondent. This opens up a type of analysis that resembles a surgical airstrike as opposed to the regular army occupation of a traditional consumer survey involving dozens of questions and taking up to $30 \mathrm{~min}$ to complete. The ability to obtain quick answers to specific questions allows firms to decentralize the marketing research function within organizations and increase demand for data-savvy analysis.

The challenge in this new world of quick analysis is in integrating results across multiple studies of this type. One study might examine the prevalence of needs associated with product category and relate these needs to specific product attributes. A second study might relate attributes and associated benefits to the creation of consideration sets, and a third might relate brands considered to their evaluation and so forth. The integration of results from among these surgical studies becomes more important as marketing research analysis becomes more differentiated.

Bayesian analysis is particularly well suited for information integration through the use of hyper-parameters that explain the variation of other parameters (see [21]). Needs and wants, for example, can be integrated in the upper level of a hierarchical model by including needs as covariates in the random effects model. This bridging of variables into a common model is currently being done at elementary level and offers great potential benefit given the availability of Big Data, both from the marketplace and in augmented form with data from online and traditional surveys that provide insight into determinants of demand.

\section{Concluding Remarks}

The future will, without a doubt, be more complex than the present. Technology will give us ever-increasing access to data as life becomes documented at finer and finer levels of detail, just as it will give us increasing computing resources to allow us to master and better understand the world we are measuring. The analytic tools of the future will not be built by computer scientists or statisticians or economists or psychologists on their own, but by dedicated teams who are building tools specific to the task at hand. Automation or tactical tools that respond to the system in optimal ways, assuming that the response will not change the system, will be the simplest tools to build. The more challenging tools will be the ones that can understand how the entire system will respond and change to "optimal" actions of the individual and the ones that can understand the impact of things that have only been imagined. In all of these tool-building efforts, Bayesian methods offer a powerful tool kit for a number of important reasons. First, Bayesian methods allow for subjective judgments, which can come from experts or experiments, to be formally included in a model, allowing for a way to calibrate the utility (or decision rules) of individual agents and to allow for theory-driven priors, see Montgomery and Rossi [16]. Second, Bayesian inference allows for stochastic searches over the space of possible models, which are given a positive a priori probability. One of the best-known cases is the case of variable selection, such as the stochastic search variable selection algorithm in George and McCulloch [9]. In many ways, the challenge with analytics going forward will not be how to deal with Big Data, but how to successfully conduct Big Analytics or how to scale the search for the right level of complexity to effetely explain (and ultimately predict) Big Data. The combinatoric challenge of finding a reasonable subset(s) of a larger set of predictors is a special case of finding the right model among an extremely large set of candidate models. It is the needle in the haystack problem, a problem that only gets harder as the haystack gets larger.

The early steps towards scaling methods (Bayesian and others), to gain insights and advantages that technology is affording us, offer some guidelines for how future efforts might proceed. First, we will have to expand our horizons and search out expertise to allow us to combine our tools with tools from other disciplines, an effort that while often challenging can become rewarding as the barriers of language give way to common ideas shared across disciplines and as new insights are reached, often transforming multiple disciplines. Second, we will have to be willing to spend the intellectual effort to make sure that the best in class theories of how individuals and systems work are guiding the development and use of the tools that are created. Both of these trends point towards economics (e.g., marketing) based research that will become more team based with increased collaboration 
between academia and industry and the need for the strengthening of institutions that can help researchers address fundamental problems that will bring greater efficiency and productivity to our broader economy.

\section{References}

1. Allenby GM, Rossi PE (1999) Marketing models of consumer heterogeneity. J Econ 89:57-78

2. Bradlow ET, Hardie BGS, Fader PS (2002) Bayesian inference for the negative binomial distribution via polynomial expansions. J Comput Graph Stat 11(1):189-201

3. Chankukala S, Edwards Y, Allenby GM (2011) Identifying unmet demand. Mark Sci 30(1):61-73

4. Chipman H, George E, McCulloch R (2010) BART: Bayesian additive regression trees. Ann Appl Stat 4(1):266-298

5. Everson PJ, Bradlow ET (2002) Bayesian inference for the betabinomial distribution via polynomial expansions. J Comput Graph Stat 11(1):202-207

6. Fennell G, Allenby GM (2014) Conceptualizing and measuring prospect wants: understanding the source of brand preference. Cust Needs Solutions 1:23-39

7. Gelfand AE, Smith AFM (1990) Sampling-based approaches to calculating marginal densities. J Am Stat Assoc 85(410):398-409

8. Gupta S, Chintagunta PK (1994) On using demographic variables to determine segment membership in logit mixture models. J Mark Res $31(1)$

9. George EI, McCulloch RE (1993) Variable selection via Gibbs sampling. J Am Stat Assoc 88:881-889

10. Jordan MI, Ghahramani Z, Jaakkola TS, Saul LK (1999) An introduction to variational methods for graphical models. Mach Learn 37: $183-233$
11. Kim J, Allenby GM, Rossi PE (2002) Modeling consumer demand for variety. Mark Sci 21(3):229-250

12. Lee S, Allenby GM (2014) Modeling indivisible demand. Mark Sci. doi:10.1287/mksc.2013.0829

13. Lee TY, Bradlow ET (2011) Automatic marketing research using online customer reviews. J Mark Res 48(5):881-894

14. McFadden D (1973) Conditional logit analysis of qualitative choice behavior. In: Zarembka P (ed) Frontiers in economics, 1973. Academic, New York

15. Miller SJ, Bradlow ET, Dayartna K (2006) Closed-form Bayesian inferences for the logit model via polynomial expansions. Quant Mark Econ 4(2): 173-206

16. Montgomery AL, Rossi PE (1999) Estimating price elasticities with theory-based priors. J Mark Res 413-423

17. Netzer O, Lattin JM, Srinivasan V (2008) A hidden Markov model of customer relationship dynamics. Mark Sci 27(2):185-204

18. Otter T, Gilbride TJ, Allenby GM (2011) Testing models of strategic behavior characterized by conditional likelihoods. Mark Sci 30(4): 686-701

19. Pratola MT, Chipman H, Gattiker DM, Higdon DM, McCulloch R, Rust W (2014) Parallel Bayesian additive regression trees. J Comput Graph Stat. doi:10.1214/09-AOAS285

20. Rockova V, George EI (2014) EMVS: the EM approach to Bayesian variable selection. J Am Stat Assoc. doi:10.1080/01621459.2013. 869223

21. Rossi PE, Allenby GM, McCulloch R (2005) Bayesian statistics and marketing. Wiley, Chichester

22. Scott SL, Blocker AW, Bonassi FV, Chipman H, George E, McCulloch R (2014) Bayes and big data: the consensus Monte Carlo algorithm. Working Paper

23. Sutton AJ, Abrams KR (2001) Bayesian methods in metaanalysis and evidence synthesis. Stat Methods Med Res 10(4):277-303

24. Tibbits MM, Haran M, Liechty JC (2011) Parallel multivariate slice sampling. Stat Comput 21(3):415-430 